\section{ISSUES}

No. 1/ February: $1-60$

No. 2/ April: $61-128$

No. 3/ June: $129-188$

No. 4/ August: $189-256$

No. 5/ October: $257-320$

No. 6/ December: $321-452$

\section{AUTHORS}

Alemdag, I. S. 87

Anderson, H. W. 195

Andresen, J. W. 291

Armson, K. A. 447

Blais, J. R. 71

Boivin, J. L. 341

Bowdery, L. 83

Burkhart, H. E. 325

Burridge, L.O.W. 287

Calvert, R. F. 226

Carlson, L. W. 147

Carroll, M. R. 8, 271

Chisholm, H. R. 447

Crown, M. 134

Fisher, R. F. 328

Flann, I. B. 219

Foster, N. W. 142
Good, W. S. 328

Gray, J. A. 313

Harper, R. 4

Hellum, A. K. 159

Hirvonen, H. E. 275

Horton, K. W. 278

Jaciw, P. 195

Jackson, D. H. 33

Jones, A. R. C. 281

Jorgensen, E. 267

Ketcheson, D. E. 336

Khalil, M. A. K. 150

Klinka, K. 348

MacArthur, J. D. 281

MacLeod, D. 77

Manning, G. H. 155

Martell, A. M. 96

McKeen, K. A. 448

Mead, D. A. 353

Morrison, I. K. 142

Morsink, W. A. G. 287

Mullin, R. E. 83

Murray, W. G. 164

Nairn, L. D. 147

Nautiyal, J. C. 20

Payandeh, B. 355
Pollard, D. F. W. 63, 294

Radvanyi, A. 96, 139

Ritcey, D. E. 159

Roberge, M. R. 223

Robitaille, L. 201

Routledge, H. T. 448

Saunders, G. L. 4

Schneider, M. H. 215

Skoda, L. 348

Smith, J H. G. 15, 91

Smyth, J. H. 332, 336

Sullivan, A. D. 325

Swan, H. S. D. 142

Teskey, A. G. 332

Thomas, R. 134

von Althen, F. W. 166, 209

Waterland, T. M. 438

Weetman, G. F. 262

Wetton, E. A. F. 26

Wilson, D. A. 448

Winston, D. A. 142

Young, H. E. 204

Young, W. 445

Zimmerman, A. 441

Zsuffa, L. 195

\title{
REGULAR FEATURES
}

Forum: 2, 62, 130, 190, 258, 322

The CIF Scene: $37,100,169,230,298$, 364

Of Interest: $41,116,184,245,312,377$

Section News: 48, 110, 180, 239, 315, 373

Book Reviews: 52, 118, 185, 247, 317, 379

\section{CONTENTS}

77 Accuracy of regeneration stocking estimates: Tests with simulated data.

271 Amenity forestry and the economic behaviour of land owners.

385 Annual meeting Vancouver. The 69th.

353 Aspen - The ugly duckling.

325 Communications among researchers and practitioners.

71 Comparative appraisal of insecticidal operations against spruce budworm in Quebec during two outbreaks.

159 Computerized reforestation records for Alberta.

96 Control of small mammals on a hardwood plantation by poison-bait feeders.

134 Cowichan valley demonstration forest, The

448 Demand and marketing, lumber and panel.

448 Demand outlook for Canadian pulp and paper.

63 Ecotour - A cross section of Canada.

139 Effects of four years storage on germination of R-55 repellent-treated white spruce seeds.

83 Effects of seedbed density and nursery fertilization on survival and growth of white spruce.
215 Energy from forest biomass.

155 Evaluating public forestry investments in British Columbia: The choice of discount rates.

332 Forest industry in west-central Canada, 1972.

20 Forest tenure structure in Ontario.

4 Forestry course for teachers: A partial answer to the communication and credibility gap. A

87 Formulation of stand volume increments in singleexamination sample plots.

209 Hardwood planting in Ontario.

204 Hardwoods within the complete forest concept.

294 Impressions of urban forestry in China.

447 Intensive forest management in the boreal forest.

447 Intensive forestry on the west coast.

448 Intensive forestry on the east coast.

441 Is our communication effort working?

336 Labor: The key to Ontario's silvicultural program.

355 Making the most of forest managers' knowledge in choosing economically desirable regeneration systems.

328 Mapping stand structure for woodlot management.

447 Maximizing fibre values.

281 Morgan arboretum - A model urban forest. 
445 National forest policy for Canada, A

438 New era in Canadian forestry, A

278 Of trees, natural ecosystems and community design.

219 Opportunities in hardwood utilization.

262 Policemen, planners and managers, in New Brunswick crown forests.

150 Provenance experiments on a sitka spruce in Newfoundland.

201 Recherches sylvicoles sur les feuillus nordiques à la station forestière de duchesnay.

341 Régénération après coupes mécanisées et conventionnelles: côte-nord du Québec.

223 Results of research in northern hardwood silviculture at Dudswell.

275 Role of urban forestry in regional landscape design, The

147 Root deformities in some container-grown jack pine in southeastern Manitoba.
8 Some aspects of the economic nature of contracts as they relate to forest land tenure in Alberta.

33 Some structural components of contracts as they relate to Canadian forest tenure.

348 Synecological map of the university of British Columbia research forest.

142 Ten-year growth in two fertilization experiments in a semimature jack pine stand in northwestern Ontario.

91 Tree-ring analyses can be improved by measurement of component widths and densities.

195 Trends and prospects in Ontario's poplar plantation management.

226 Tree improvement and hardwood silviculture.

287 Urban forestry in Windsor, Ontario.

291 Urban forestry legislation in Ontario.

26 Variations in implementing sustained-yield forestry in Canada - British Columbia.

15 Variations in implementing sustained-yield forestry in Quebec.

267 Vegetation needs and concerns in urban areas.

\section{$\square$ Alan Moss \& Associates Ltd.}

Consultants in Forestry and Forest Industries

\section{Domestic and International}

1420 St. Paul Street

Kelowna, British Columbia V1Y 2E6

Telephone: (604) 763-4811

Cable: Sylva

\section{Woodlot Service Ltd.}

"All Matters Perfaining to Forestry"

GORDON B. YOUNG, B.Sc.F., M.F.

Registered Professional Forester

320 Maple Street

Bus.: $506-472-3396$

Fredericton, N.B.

Home: 506 - 472-7721

\section{FORESTRY CONSULTANTS INC.}

115 N. CUMBERLAND STREET THUNDER BAY, ONTARIO

P7A 4M3

TEL.: 344-0811

\author{
RESOURCE INVENTORY - \\ FOREST MANAGEMENT PLANNING \\ SILVICULTURAL SPECIALISTS \\ SYSTEM DESIGN AND CONTRACT
}

\section{EDWARD}

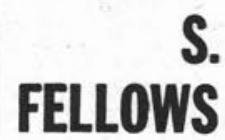

FORESTRY \& FOREST PRODUCTS CONSULTANT

P.O. Box 354, 404 Queen St., FREDERICTON, N. B.

Registered Professional Forester (N. B.)

MEMBER:

Canadian Institute of Forestry

Forest Products Reseorch Society, Etc.

INDUSTRY DEVELOPMENT - FOREST PRODUCTS ECONOMIC FOREST POLICY \& ADMINISTRATION 


\section{Canadian Institute of Forestry - Institut Forestier du Canada}

Incorporated 1950

Founded 1908

1977-78 National Officers

PRESIDENT: P.E. Boucher, 498 York St., Fredericton, N.B. E3B 3P7

1st VICE PRESIDENT: D.R. Redmond, 643 Tillbury Avenue, Ottawa, Ont. K2A OZ9

2nd VICE PRESIDENT: J.H.G. Smith, Faculty of Forestry, University of British Columbia, Vancouver, B.C. V6T 1W5
PAST PRESIDENT: A.B. Anderson, 2547 Ottawa Street, W. Vancouver, B.C. V7V 2T3

EDITOR: J.D. MacArthur, Box 5000, Macdonald College, P.Q. HOA 1 CO.

SECRETARY-MANAGER: A. G. Racey, Box 5000, Macdonald College, P.Q. HOA $1 \mathrm{CO}$.

\section{Section Representatives}

ALGONQUIN: I. R. Methven, Petawawa F.E.S., Chalk River, Ont.

CARIBOO: R. W. Baker, 1021 François Cres., Prince George, B.C.

CENTRAL ONTARIO: B. J. Stocks, Box 387, Sault Ste. Marie, Ont. P6A 5M1.

CHAMPLAIN: G. Swant, Forestry Dept., Kirkland Campus, John Abbott College, Kirkland, P.Q. H9H 3L4.

KOOTENAY: J.L. Adams, 1909-4th Ave. S., Castlegar, B.C. V1N 2T5.

LAKE OF THE WOODS: F.S. Harrison, 22 Eagle Drive, Dryden, Ont. P8N 2C9

MANITOBA: N. B. Tuinhof, 31 Hammond Rd., Winnipeg, Man. R3R 1L9.

MARITIME: N. L. Kissick, 792 Borden St., Fredericton, N.B. E3B 4C4

NEWFOUNDLAND: J. A. Cook, Faculty Applied Science, Memorial University of Newfoundland, St. John's, Nfld.

NORTHERN ONTARIO: V.I. Sleep, 113 Riverside Dr., Kapuskasing, Ont. P5M 1B4

NORTHWESTERN ONTARIO: Gary R. Bloomfield, 435 Simon Fraser Dr., Thunder Bay, Ont. P7C 4 Z9.

\section{on the Board of Directors}

NOVA SCOTIA: C. A. Steel, Dept. of Lands and Forests, Box 698, Halifax, N.S. B3J 2 T9.

OKANAGAN: S. N.McLean, 171 Wilson St., Kamloops, B.C. V2B 3G9.

ORLEANS: L. LeBlanc, 863 Cherbourg, Ste. Foy, P.Q. G1X $2 W 8$.

OTTAWA VALLEY: E. Jorgensen, The Watergate No. 1714, 211 Wurtenburg St., Ottawa, Ont. K1N 8R4.

PACIFIC: W. E. Dumont, P.O. Box 4000, Port Alice, B.C. VON 2 NO.

ROCKY MOUNTAIN: J. A. Beck, Jr., Dept. of Forest Science, University of Alberta, Edmonton, Alta. T6G 2E1.

SAGUENAY: R. N. Ouellet, The Price Co. Ltd., P.O. Box 68, Chicoutimi, P.Q. G7H 5B6

SASKATCHEWAN: A. Kabzems, 205-9th St. E., Prince Albert, Sask. S4T 6M7.

SOUTHERN ONTARIO: D.E. Barron, 124 Lakeshore Road, St. Catharines, Ont. L2N 2T5.

VANCOUVER: J. W. Toovey, 2976 Williams Ave., N. Vancouver, B.C. V7K 1 Z6.

VANCOUVER ISLAND: J. N. MacFarlane, 2475 Selne Rd. Duncan, B.C.

\section{SECTION OFFICERS}

\section{Chairman}

ALgONQUiN: W. H. Collins, 358 Broadview, Pembroke, Ont. Ont. K8A $2 \mathrm{~A} 8$.

CARIBOO: F.J. Lowenberger, Industrial Forestry Service, \#101 - 1595 Fifth Ave., Prince George, B.C. V2L 3L9.

CENTRAL ONTARIO: B.J. Stocks, Box 387, Sault Ste. Marie, Ont. P6A $5 \mathrm{M} 1$.

CHAMPLAIN: P. M. Morley, 3145 The Boulevard, Montreal, P.Q. H3Y $1 \mathrm{~S} 1$

KOOTENAY: J.R. Cuthbert, 912 Kokanee St., Nelson, B.C. V1L 3P8.

LAKE OF THE WOODS: G. Brown, $716-6$ th St. S., Kenora, Ont. P9N $1 \mathrm{~N} 8$

MANITOBA: J.R. Hreno, 860 Lyon St., Ft. Garry, Winnipeg, Man. R3T 0G7.

MARITIME: H. Oldham, Maritime Forest Research Centre, CFS, Box 4000, Fredericton, N.B.

NEWFOUNDLAND: E. Blackmore, 17 Peterson Dr., Gander, Nfld.

NORTHERN ONTARIO: V.I. Sleep, 113 Riverside Dr., Kapuskasing, Ont. P5M $1 \mathrm{~B} 4$.

NORTHWESTERN ONTARIO: D.J. Richardson, School of Forestry, Lakehead University, Thunder Bay, Ont.

NOVA SCOTIA: E.S. Atkins, Dept. of Lands \& Forests, Dennis Bldg., Halifax, N.S.

OKANAGAN: I.S. Tolnai, Weyerhaeuser Canada Ltd., Box 800 , Kamloops, B.C. V2C $5 \mathrm{M7}$

ORLEANS: Lise Robitaille, Service de la Recherche, Min. des Terres et Forêts, 2700 Einstein, Ste Foy, P.Q. G1P 3W8

OTTAWA VALLEY: R. H. Newnham, Forest Management Inst. Environment Canada, 240 Bank St., Ottawa, Ont. K1G 3 Z6

PACIFIC: E. R. Mattice, $830-13$ th Ave., Campbell River, B.C. V9W $4 \mathrm{H} 2$.

ROCKY MOUNTAIN: C. E. Andersen, Box 254, Grande Prairie, Alta. T8V $3 A 4$.

SAGUENAY: To be named.

SASKATCHEWAN: V.G. Begrand, Box 1241, Hudson Bay, Sask. SOE OYO.

SOUTHERN ONTARIO: R. Grinnell, 86 Truman Rd., Willowdale, Ont. M2L 2 L6.

VANCOUVER: P. W. Ackhurst, 3950 Sunset Blvd., N. Vancouver, B.C. V7R $3 \times 9$.

VANCOUVER ISLAND: M. D. Meagher, R.R. \#5, Tzouhalem Rd., Duncan, B.C. V9L $1 \mathrm{M} 3$

\section{Secretary}

ALGONQUIN: A. B. Berry, Petawawa Forest Experiment Station, Chalk River, Ont. KOJ 1J0.

CARIBOO: N. B. Crist, 4347 Horsefly Ave., Prince George, B.C. V2M 5C4.

CENTRAL ONTARIO: G. D. Huntley, Box 387, Sault Ste. Marie, Ont. P6A 5M1.

CHAMPLAIN: G. Swant, Forestry Dept., Kirkland Campus, John Abbott College, Kirkland, P.Q. H9H 3L4.

KOOTENAY: D. O. Parminter, 518 Lake St., Nelson, B.C. V1L 4C6.

LAKE OF THE WOODS: A.M. Clark, 401-7th Ave. N., Kenora, Ont. P9N 3J2.

MANITOBA: L. G. Yarn, 4272 Eldridge Ave., Winnipeg, Man. R3R 0L7.

MARITIME: J. Torunski, Maritime Forest Ranger School, R.R. \#5, Fredericton, N.B. E3B 4X6.

NEWFOUNDLAND: J.A. Taylor, 5 Grey Ave. Ext., Gander, Nfld. A1V $1 Z 4$

NORTHERN ONTARIO: R. G. Lightheart, 9 Maple Dr., Kapuskasing, Ont. P5N $2 A 5$.

NORTHW'ESTERN ONTARIO: K. M. McClain, 426 Ambrose St., Thunder Bay, Ont. P7B $1 \mathrm{M} 6$.

NOVA SCOTIA: C. A. Steel, Dept. of Lands \& Forests, Box 698, Halifax, N.S. B3J 2T9.

OKANAGAN: R.G. Jennejohn, c/o District Forester, B.C. Forest Service, Kamloops, B.C. V2C 2T7.

ORLEANS: E. Rey-Lescure, Centre Recherches Forestières des Laurentides, C.P. 3800, Ste. Foy, P.Q.

OTTAWA VALLEY: E. Stechishen, Box 893, Manotick, Ont. KOA 2 NO.

PACIFIC: D. K. Haley, Box 543, Port McNeil, B.C. VoN 2RO. ROCKY MOUNTAIN: J. Bauer, 7810 Patterson Dr., Grande Prairie, Alta. T8V 3R7.

SAGUENAY: H. W. Journeaux, 306 Foucault Ave., Arvida, P.Q. G7S 2 L2.

SASKATCHEWAN: H.S. Price, 359-21st Street W., Prince Albert, Sask. S6V 4J2.

SOUTHERN ONTARIO: Wm. D. Baker, 740 Windermere St., Toronto, Ont. M6S 3M3

VANCOUVER: D. E. Shaw, c/o L \& K Lumber, Box 86219, N. Vancouver, B.C. V7L $4 \mathrm{~K} 2$.

VANCOUVER ISLAND: P.K. Diggle, 2784 Foul Bay Rd., Victoria, B.C. V8R $5 \mathrm{C} 2$. 